\title{
A Methodological Survey and Proposed Algorithm on Image Segmentation using Genetic Algorithm
}

\author{
Megha Agarwal \\ Computer Science Department \\ IMS Engineering College, Ghaziabad, U.P., India
}

\author{
Vijai Singh \\ Asst. Prof. Computer Science Department \\ IMS Engineering College, Ghaziabad, U.P., India
}

\begin{abstract}
This literature review attempts to provide a brief overview of some of the most common image segmentation techniques. It discusses Edge detection technique, Thresholding technique, Region growing based technique, Watershed technique, Compression based method, Histogram based segmentation and Graph partitioning method. With the growing research on image segmentation, it has become important to categorise the research outcomes and provide readers with an overview of the existing segmentation techniques in each category. In this paper different method of implementing genetic algorithm has been reviewed. Finally, summaries and review of research work on wrapper approach for image segmentation techniques has been represented.
\end{abstract}

\section{General Terms}

Image processing, image segmentation, image classification, genetic algorithm, wrapper approach, object detection.

\section{Keywords}

Color image, Genetic algorithm, segmentation techniques, wrapper approach.

\section{INTRODUCTION}

Image segmentation is the process of separating or grouping an image into different parts. There are currently many different ways of performing image segmentation, ranging from the simple thresholding method to advanced color image segmentation methods. These parts normally correspond to something that humans can easily separate and view as individual objects. Computers have no means of intelligently recognizing objects, and so many different methods have been developed in order to segment images. The segmentation process in based on various features found in the image. This might be color information, boundaries or segment of an image. The aim of image segmentation is the domainindependent partition of the image into a set of regions, which are visually distinct and uniform with respect to some property, such as grey level, texture or colour. Segmentation can be considered the first step and key issue in object recognition, scene understanding and image understanding.

The segmentation problem is formulated as an optimization problem and Genetic Algorithm efficiently locate the global maximum in a search space and solves the problem of parameter selection in image segmentation. The aim of this paper is to review Genetic Algorithm applications for image segmentation using wrapper approach.

\section{IMAGE METHODOLOGIES}

\subsection{Edge Detection Technique}

Edge detection technique is an image segmentation technique that determines the presence of an edge or line in an image and outlines them in an appropriate way [1]. Generally, an edge is defined as the boundary pixels that connect two separate regions with changing image amplitude attributes such as different constant luminance and tristimulus values in an image [1][10][3]. This process detects outlines of an object and boundaries between objects and the background in the image. The main purpose of edge detection is to simplify the image data in order to minimize the amount of data to be processed [2]. The detection operation begins with the examination of the local discontinuity at each pixel element in an image. Amplitude, orientation, and location of a particular subarea in the image that is of interest are essentially important characteristics of possible edges [1]. Based on these characteristics, the detector has to decide whether each of the examined pixels is an edge or not. Salem Saleh Al-amri et. al.[40] implemented the edge detection segments using (MATLAB R2007a, 7.4a) and tested one satellite image (Saturn) illustrated in the Figure 1. The sobel edge detection operator is a discrete differentiation operator. It has a better noise suppression feature that makes it preferable over prewitt operator. The prewitt edge detector [40] estimates the magnitude and orientation of an edge. It is limited to 8 possible orientations and each direction orientation is a $3 \mathrm{X} 3$ neighbourhood mask. The Canny algorithm is an optimal edge detector algorithm that marks as many actual edges as possible and an edge is marked only once. Canny technique to find edges does not create false edges due to the presence of noise i.e. it isolates noise [40] from the image before finding edges of image, without affecting the features of the edges in the image and then applying the tendency to find the edges and the critical value for threshold. The Laplacian is usually used to establish whether a pixel is on the dark or light side of an edge. The Roberts Cross [40] operator performs a simple, quick to compute, 2-D spatial gradient measurement on an image. It is used mainly when diagonal edges are of main interest. Frei and Chen [1] suggest that edge detection is best carried out by simple edge detector, followed by a morphological thinning and linking process to optimize the boundaries. They gave an overview of first and second order derivative edge detections, edge fitting detection model as well as the detector performance evaluation, and also the three most frequently used edge detection methods are used are, Roberts Edge Detection, Sobel Edge Detection and Prewitt edge detection [21]. 


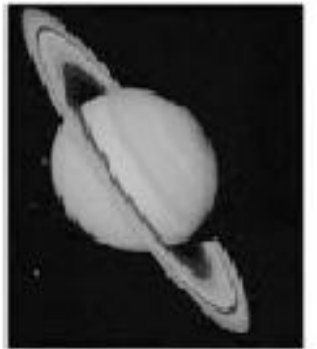

Original

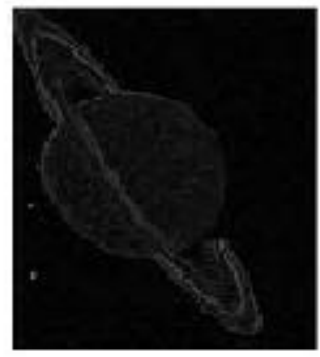

Roberts

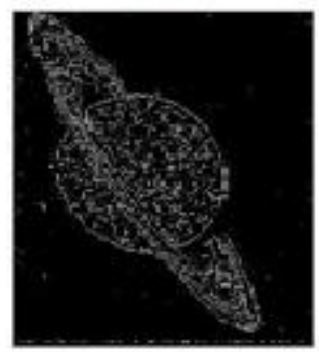

Laplacian

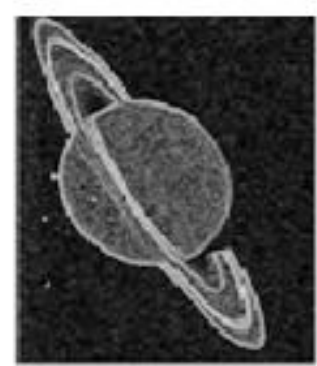

Sobel

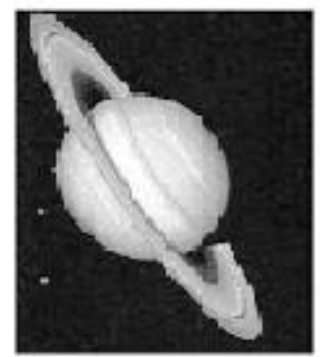

Prewitt

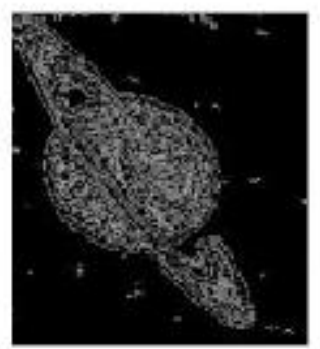

Canny
Fig 1: Satellite image (Saturn)

In the recent research, a paper proposed the implementation of a very simple but efficient fuzzy logic based algorithm to detect the edges of an image without determining the threshold value. The proposed approach begins by scanning the images using floating $3 \times 3$ pixel window. Fuzzy inference system designed has 8 inputs, which corresponds to 8 pixels of instantaneous scanning matrix, one output that tells whether the pixel under consideration is "black", "white" or "edge" pixel. Rule base comprises of sixteen rules, which classify the target pixel [28]. The proposed method results for different captured images are compared to those obtained with the linear Sobel operator.

\subsection{Thresholding Technique}

Thresholding is one of the simplest method of image segmentation. From a gray scale image, thresholding can be used to create binary images (Shapiro, et al 2001:83). Threshold segmentation techniques can be grouped in three different classes:

1. Local techniques are based on the local properties of the pixels and their neighbourhoods. In local thresholding, the original image is divided into smaller non-overlapping subimages using a threshold that is determined for each of these sub-images. This yields a thresholded image with gray level discontinuities at the boundaries of two different sub-images.
The threshold of a region can be determined by either the point-dependent method or the region-dependent method. A smoothing technique, if required, is then applied to eliminate the discontinuities. Chow and Kaneko [41, 42] suggest the use of a 7 X 7 window for local thresholding. In their method, the original image is divided into $7 \times 7$ sub-images and a threshold is computed for each sub-image.

2. Global techniques segment an image on the basis of information obtain globally (e.g. by using image histogram; global texture properties). Mason et al. [43] proposed the use of an edge operator (e.g., the Laplacian, the Roberts cross, etc.) for weighting. According to their method, the values of the edge operator are small for pixels in homogeneous regions and these pixels are given more weight. However, the values of the edge operator are large for pixels in the neighbourhood of an edge and these pixels are given less weight. The weight can be computed as $1 /\left(1+\Delta^{2}\right)$, where $\Delta$ is the edge value at a given pixel. As a result of this weighting process, the new gray level histogram will have sharper peaks and deeper valleys.

In 1965, Katz [44] considered that since the pixels in the neighbourhood of an edge have higher edge values, the gray level histogram for these pixels should have a single peak at a gray level between the object and the background gray levels. This gray level is, therefore, a suitable choice of the threshold value. This has also been studied by Weska and Rosenfeld [45]. Several variations of the above method have been proposed [46,47,48] Weszka and Rosenfeld [49] unified them.

3. Split, merge and growing techniques use both the notions of homogeneity and geometrical proximity in order to obtain good segmentation results. Finally image segmentation, a field of image analysis, is used to group pixels into regions to determine an image's composition [12][13].

Fuzzy C-means, its improvement methods algorithm and strategies for remote sensing image segmentation can offer less iterations times to converge to global optimal solution. At the same time, it has good stability and robustness. Its good effect of segmentation can improve accuracy and efficiency of remote sensing image threshold segmentation [23]. Color images can also be thresholded. N. Senthilkumaran, R. Rajesh [21] proposed to designate a separate threshold for each of the RGB components of the image and then combine them with an AND operation. This reflects the way the camera works and how the data is stored in the computer, but it does not correspond to the way that people recognize color. Therefore, the HSL and HSV color models are more often used. It is also possible to use the CMYK color model (Pham et al., 2007).

\subsection{Region Growing Based Technique}

Region growing is based on selecting initial seed points and adding neighbouring pixels to the region depending on the suitable membership criteria such as colour similarity. Initially seed regions are identified based on calculation of variance of $\mathrm{R}, \mathrm{G}$ and $\mathrm{B}$ components and selecting the first seed point having the minimum R, G, B variance product. 4 connected neighbours are used to grow from the seed point. This process [24] is an iterative procedure and runs until no change occurs in two successive stages. The major drawback of the algorithm is that it segments the image based only on colour/intensity, hence it gives poor results for textured image [24]. Also, it depends largely on the value of $\mathrm{T}$ in likelihood term computation which needs to be taken different value for different images. 
Rolf Adams and Leanne Bischof [50] presented a new method known as seeded region growing (SRG) that uses the conventional homogeneity principle of region growing technique as its base with its mechanism close to the watershed technique. This technique uses a set of points, known as seeds that are grouped together into $\mathrm{n}$ sets which serve as a beginning of the SRG algorithm. Sometimes each set is comprised of single points instead of a group of points. SRG then segments the image into regions with the property that each connected component of a region meets exactly one of the seeds and hence the regions that are as homogeneous as possible are selected. The requirement to obtain a good segmentation in case of SRG is that, if the regions are relatively noiseless, each seed pixel has a gray value typical of its region. However, if the regions are noisy consider using small seed areas, instead of single pixel, so that a proper estimate of the region's mean is acquired.

Region Growing can also be done by Mean or Max-Min strategies [27]. The image is first partitioned into uniform regions for processing. Region growing is executed to merge the adjacent regions which meet the specified criteria. This criteria could be either max-min difference or mean value of each region.

The max-min and the mean algorithms [27] each posed some problems with various image features. Former preserved edges and handled some textures better while the latter did better on disfigured images. Thus the selection of any of these algorithms depends on the input image which is to be worked upon. Neither of these algorithms performs high level spatial correlation, so they fail to work on complex images consisting of complex regions.

\subsection{Watershed Technique}

The watershed transform [5] is a popular segmentation method coming from the field of mathematical morphology. Mathematical morphology [5] is a collection of operators based on set theory and defined on an abstract structure known as an infinite lattice. The mathematical morphology is intended to analyze and recognize the geometrical properties and structure of the objects. Such analysis must be quantitative in order to provide a mathematical framework for describing the spatial organization. The intuitive description of the watershed transform is simple: considering the image as a topographic relief, where the height of each point is directly related to its gray level, and considering rain gradually falling on the terrain, then the watersheds are the lines that separate the "lakes" (actually called catchments basins) that form. Generally, the watershed transform is computed on the gradient of the original image, so that the catchments basin boundaries are located at high gradient points.

As an interpretation in topography in [51] Sridevi et. al. mentioned that the watershed can be imagined as the high mountain that separates two regions. Each region has its own minimum and, if a drop of water falls on one side of the watershed, it will reach the minimum of the regions. Points of contact between the propagation originating from different minima are defined as the boundaries of the regions and are used to create the final partition. A common difficulty in measuring the images occurs when features touch, and therefore cannot be separately identified, counted, or measured. This situation may arise where actual feature may overlap or when the particles resting on a surface tend to agglomerate and touch each other. One method of separating touching but most convex features in an image is called watershed segmentation. The regions that the watershed separates are called catchment basins. Lines separating catchment basins are watershed lines and are interpreted as boundaries between regions for the purposes of segmentation $[52,53]$.

The concept of watersheds is based on visualizing an image in three dimensions: two spatial coordinates versus grey levels. In such a topographic interpretation, we consider three types of points as follows:

1. Points belonging to regional minimum.

2. Points at which water drops.

3. Points at which water would be equally likely to fall.

For a particular regional minimum, the set of points satisfying condition (2) is called catchment basin or watershed of that minimum. A watershed region or catchment basin is defined as the region over which all points flow "downhill" to a common point. The points satisfying condition (3) are termed divide lines or watershed lines (Gonzalez and Woods, 2008). Watersheds are one of the classic regions in the field of topography. Watershed use image gradient to initial point and region can obtain by region growing. The accumulation of water in the vicinity of local minima is called a catchment basin. There are two approaches to find watershed of an image.

i. Rainfall approach.

ii. Flooding approach.

In rainfall approach, local minima are found throughout the image, and each local minimum is assigned unique tag. A conceptual water drop is placed at each untagged pixel. The drop moves to low amplitude neighbour until it reaches a tagged pixel and it assumes tag value.

In flooding approach, conceptual pixel holes are pierced at each local minimum. The water enters the holes and proceeds to fill each catchment basin. If the basin is about to overflow, a dam is built on its surrounding ridge line to the height of high altitude ridge point. These dam boundaries correspond to the watershed lines [54].

Steps in Watershed Algorithm as follows,

1. Read in an Image and covert it in gray scale.

2. Use the gradient magnitude as the segmentation function.

3. Mark the foreground objects.

4. Compute the Background markers.

5. Compute the watershed transform of the segmentation function.

6. Visualize the result.

Due to the numerous advantages of watershed transform, it has been widely used in many fields of image processing, including medical image segmentation, it is fast and can be parallelized (in [7], an almost linear speedup was reported for a number of processors up to 64), and it produces a complete division of the image in separated regions even if the contrast is poor, thus avoiding the need for any kind of contour joining. Several researchers have proposed techniques to embed the watershed transform in a multi scale framework, thus providing the advantages of these representations [94].The watershed transform has interesting properties that make it useful for many different image segmentation 
applications: it is simple and intuitive, can be parallelized, and always produces a complete division of the image[25].

\subsection{Histogram based Segmentation}

This approach is based on the assumption that objects within each class should have high degree of similarity, while those in different classes should be dissimilar. The histogram retains a uniform colour transition that enables us to do a windowbased smoothing during retrieval. The clusters are found by mode analysis of the multidimensional histogram of the considered vectors through a non-iterative peak-climbing approach [20]. Among all segmentation techniques, the automatic thresholding methods are widely used because of their advantages of simple implement and time saving. Otsu method is one of thresholding methods and frequently used in various fields.

\subsection{Graph Partitioning Method}

Spectral graph partitioning provides a powerful approach to image segmentation. This approach produces the high quality segmentations of spectral methods, but with improved speed and stability [16]. Many graph theoretic techniques have been proposed for cluster analysis. Commonly known techniques are- single- link and complete link hierarchical algorithms implemented using a threshold graph, forming clusters by breaking inconsistent arcs in the minimum spanning tree of the proximity graph, detecting clusters using directed trees [31]. This segmentation approach is able to accurately locate region boundaries, and at the same time guarantees the formation of closed edge contours.

\subsection{Compression Based Methods}

The main idea behind compression based methods [26] is that the optimal segmentation is the one that minimizes, over all possible segmentations, the coding length of the data [75][76]. The concepts of the optimal segmentation and the coding length are inter-related in the way that segmentation is done in a manner so that the regularities of the pattern, if occurring in an image, can be successfully compressed. Two features of the image are used by this method: boundary and texture. Each of these components is modelled by a probability distribution function and its coding length is evaluated. Firstly, the boundary of the regions in natural images tends to have a smooth form. Thus the smoother a boundary, the shorter coding length will be. Secondly, the texture is compressed similar to minimum description length (MDL) principle. The texture in each region is modelled by a multivariate normal distribution. The estimated entropy [75][76] bounds the true entropy of the data from above. Therefore the true coding length is not more than the objective of the algorithm. This scheme yields the number of bits required to encode that image based on the given segmentation. Thus, among all possible segmentations of an image, the goal is to find such a segmentation which produces the shortest coding length. This can be achieved by a simple agglomerative clustering method. The distortion in the lossy compression determines the coarseness of the segmentation and its optimal value may differ for each image. This parameter can be estimated heuristically from the contrast of textures in an image.

Compression-based methods are also very helpful in cases of storage limitation. Recently a hybrid image compression method is proposed [77]. In the mentioned paper the image is segmented into background and foreground information. The foreground of the image is compressed at a higher quality level than the background of the image. For compression edge based segmentation is done. In order to preserve the essential parts of the given image the proposed method adapts variable quantization and threshold values corresponding to background and foreground. This hybrid approach increases the compression ratio and produces a desired high quality compressed image.

\section{BENCHMARKING SEGMENTATION}

Comaniciu and Meer (2002), describe a segmentation method based on the mean-shift algorithm. The mean-shift algorithm (Cheng 1995) [38] is designed to locate points of locally maximal density in feature space. Feature vectors containing grey-scale or colour information as well as pixel coordinates are computed for each pixel. Then the algorithm looks at a local neighbourhood in feature space centred at each pixel's feature vector and follows an iterative procedure until a convergence condition is satisfied. The overall testing procedure is as follows: for each combination of parameters, the algorithm is run over the complete set of 300 images from the BSD (Berkeley Segmentation Database) [38]. Here the curve corresponds to a spatial bandwidth $S B=8$, the precision parameter and the recall parameter are 1. Entropy consideration measures the disorder in the system. Here we use 5 values, i.e., eigenvectors for merging the threshold between $[.0625, .75]$, and for each of these we compute a tuning curve that corresponds to variations of $d$ within $(5,40)$. The tuning curves describe the behaviour of the algorithms as input parameters change. Over-segmentation is characterized by high recall but low precision, and that under-segmented images correspond to high precision, but low recall.

\section{GENETIC ALGORITHM}

Genetic Algorithms were invented to mimic some of the processes observed in natural evolution to solve the optimization problems. Genetic algorithms (GAs) [55] are a relatively new paradigm for a search, based on principles of natural selection. As early as 1962, John Holland's work on adaptive systems laid the foundation for later developments. By 1975, a book Adaptation in Natural and Artificial Systems, by Holland, his students and colleagues was published that further encouraged the research. Early to mid-1980s, genetic algorithms were being applied to a broad range of subjects. In 1992 John Koza used genetic algorithm to evolve programs to perform certain tasks. He called his method "genetic programming" (GP). They were first introduced by John Holland in 1960s [56, 57]. Genetic algorithms are proven to be the most powerful optimization technique in a large solution space. This explains the increasing popularity of Genetic algorithms applications in image processing and other fields. They are used where exhaustive search for solution is expensive in terms of computation time [17]. Genetic algorithms are advantageous in that they are able to forego local optima in an attempt to reach the global optimum [59]. This makes them far less likely to get caught in a local optimum than deterministic optimization techniques, such as local hill-climbing and gradient descent. Applications of GAs 
for image processing extend from evolving filters or detecting edges to making complex decisions or classifying detected features. Genetic algorithms are based on natural selection discovered by Charles Darwin [60]. They employ natural selection of fittest individuals as optimization problem solver. Since in nature competition among individuals for scanty resources results in the fittest individuals dominating over the weaker ones.

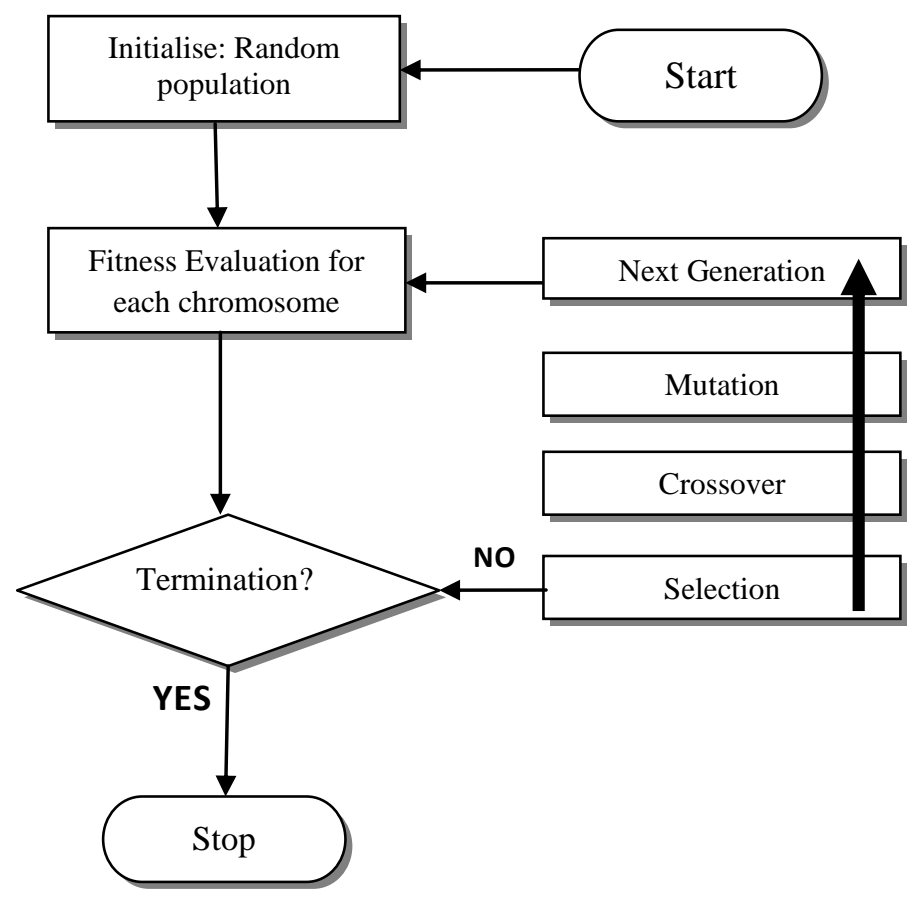

Fig 2: Flowchart of Genetic Algorithm

Optimization is performed through natural exchange of genetic material between parents. Offspring are formed from parent genes. Fitness of offspring is evaluated. The fittest individuals [9] are allowed to breed only. In computer world, genetic material is replaced by strings of bits and natural selection replaced by fitness function. Matting of parents is represented by cross-over and mutation operations [55]. Genetic algorithms are used for the optimization of relevant parameters in the existing segmentation algorithms [17, 62]. Farmer and Shugars [17] categorized the applications of genetic algorithms used for image segmentation into two major classes:

1. Parameter selection, where genetic algorithms are used to modify the parameters of an existing image segmentation method to improve its output, i.e. application to segmentation parameter selection for improved segmented outputs.

2. Pixel-level segmentation, where genetic algorithms are used to perform region labelling, i.e. application to pixel-level segmentation involving region labelling.

Most image segmentation methods have many parameters for optimization, thus the first method is used more often $[6,63$, 15]. Bhanu et al. [6] used genetic algorithms for adapting four parameters of the Phoenix segmentation algorithm [64] for outdoor color imagery. Feitosa et al. [65] modified the region growing segmentation algorithm using a fitness function based on the similarity of resulting segments to a target segmentation provided by the user. Zingaretti et al. [66] applied genetic algorithms to unsupervised color image segmentation techniques, which resort to multi-pass thresholding during each pass of the algorithm. Pignalberi et al. [67] focused on range images, by segmenting outside surfaces of 3D objects. However, this method can also be applied to segmentation of 2D images as well.

In pixel-level segmentation, genetic algorithms find use in region labelling depending on the characteristics of constituent pixels [67]. Peng et al. [58] represented each pixel in an image by a chromosome, which labels a region. Vitorino Ramos, Fernando Muge[15] applied k-Means unsupervised clustering methods into Genetic Algorithms, for finding the optimal or sub-optimal data partition, which requires a nontrivial search because of its intrinsic NP-complete nature thereby obviating any user-intervention in the segmentation process. They focussed on the demonstration of the efficiency of Genetic Algorithms to automatic and unsupervised texture segmentation [29]. Chun and Yang [68] used a fuzzy fitness function to account for the associated uncertainty in their proposed genetic algorithm based segmentation technique. Gong and Yang [69] represented the original and segmented images by means of quad-trees. They defined a two pass genetic algorithm based optimization system similar to the method by Zingaretti et al. [66]. In the first pass, genetic algorithms minimize an energy function. In the second pass, fine tuning of the segmentation method is carried out. Zingaretti et al. [66] proposed unsupervised colour image segmentation using genetic algorithm. This is another case of parameters of an existing image segmentation method being tuned by genetic algorithms. A key difference in this method is that it performs multi-pass thresholding. Different thresholds are adapted during each pass of genetic algorithms. This approach successfully segmented a wide variety of images, with the exception of images that were highly textured [8].

Pignalberi et al [67] used genetic algorithms for the optimization of parameters in an image segmentation algorithm. In this case, they focused on range images, where a pixel is coloured depending on the distance between the object and a sensor. This method segments out surfaces of 3D objects, but could be applied to segmentation of 2D images. Tianzi Jiang, Faguo Yang, Yong Fan, and David J. Evans proposed a parallel genetic algorithm [11] for cell image segmentation under severe noise. A priori knowledge about cell shape is incorporated in their method. The segmentation results of noisy human thyroid and small intestine cell images demonstrate that the proposed method is very successful in segmenting images of elliptically shaped cells. Genetic algorithms have been used to solve a wide variety of problems. In image processing [18], genetic algorithms have successfully been used for feature extraction, object recognition, knowledge based segmentation and image classification. 


\section{WRAPPER APPROACH USING GAUSSIAN ESTIMATOR}

The conventional flow of image segmentation process followed by classification assumed that the segmentation process has been able to successfully extract the object of interest. This traditional segmentation can also be viewed as a filter operating on the image independently of the classifier similar to the filter methods for feature selection. It is critical to recognize that current image segmentation algorithms are primarily open-loop in nature, as noted by Bhanu [70][71] implying that the existing segmentation algorithms are fundamentally complex spatial filters, operating "blindly" on the incoming image with no context regarding the object being segmented. It is thus an unrealistic approach to assume to obtain the required object segmented correctly without any semantic information regarding the class of objects in the scene. Hence obtained segmented map has been done correctly. M.E. Farmer and Anil K. Jain [14] proposed in their paper a closed-loop framework for image segmentation, which uses the object classification subsystem as an integral part of the segmentation process. They proposed wrapping the segmentation and the classification together and using the classifier to determine the metric for selecting the best segmentation. By using shape as the classification [72], they developed a segmentation algorithm that relaxes the feature requirement that the object of interest to be segmented must be homogeneous in some low-level image parameter, such as texture, color, or grayscale. This represents an improvement over other segmentation methods that have used classification information only to modify the segmenter parameters, since these algorithms still require an underlying homogeneity in

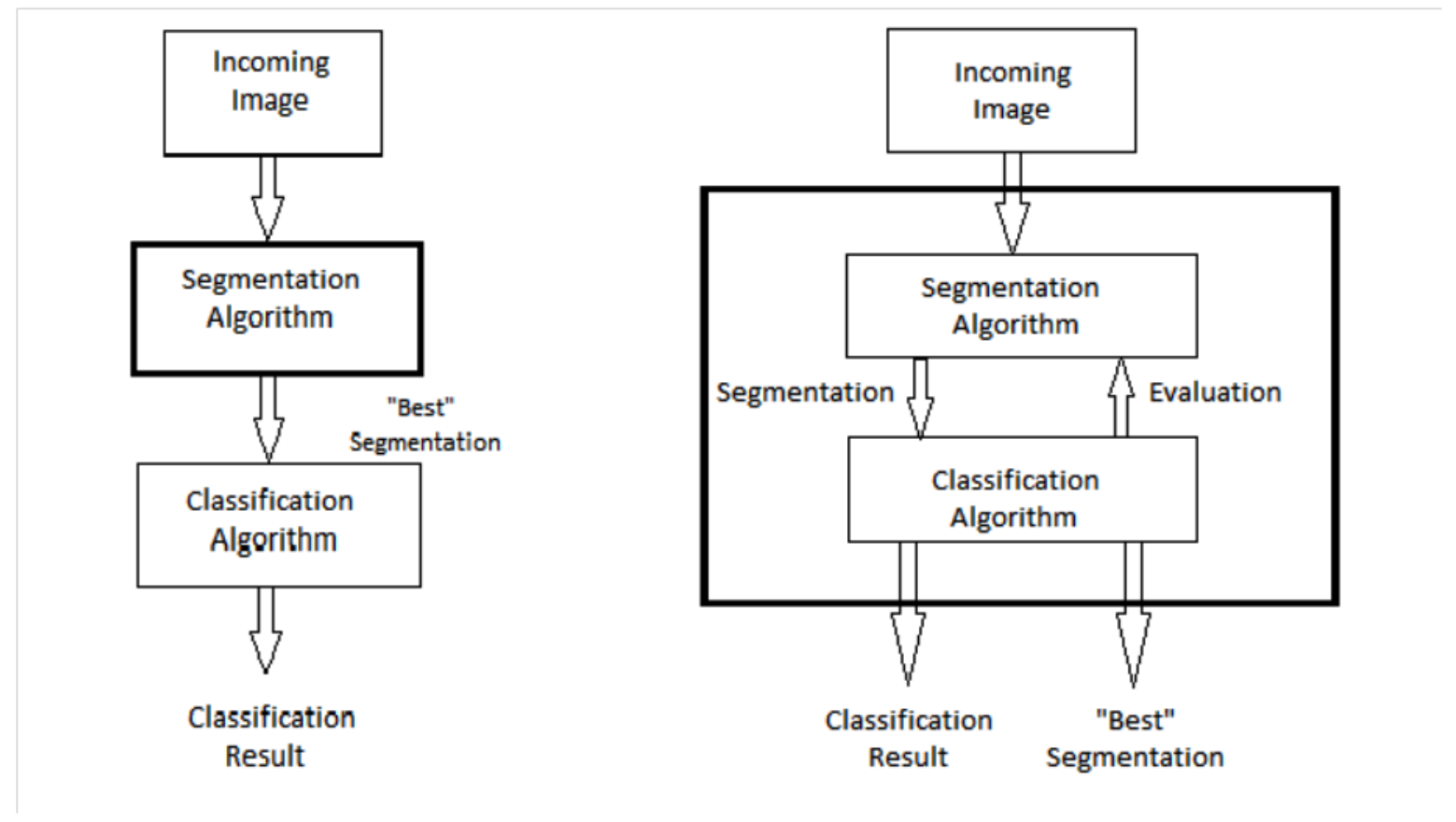

(a)

(b)

Fig3: Comparison (a) filter-based segmentation (b) wrapper-based segmentation

some parameter space. This research was extended to the applicability of wrapper to wide area surveillance of images containing possibly multiple objects of interest [22]. The approach is demonstrated on aerial images from the Katrina disaster to be able to detect buildings for possible damage assessment. In the wrapper methods for feature selection [14], the classifier is an integral part of the selection process and serves as the metric to decide the best feature set. In the same way, the segmentation and classification are wrapped together, and the classification accuracy is used as the metric to determine the best segmentation. The wrapper-based methods [74] employ some inductive classification algorithm(s) to evaluate the goodness of subset of features being selected. The performance of this approach relies on two factors: the strategy to search the space of all possible feature subsets; and the criterion to evaluate the classification accuracy of the selected subset of features. The purpose of feature selection [73] is to find the 'good' features by just selecting one representative feature subset from all features. The wrapper approaches of feature selection aim to find the minimum discriminative features to reach the high classification accuracy.

Aritz P'erez, Pedro Larra naga, and Irnaki Inza[19] proposed an approach to deal with continuous variables. They presented the continuous classifiers supported by the Gaussian network paradigm, which assumes that variables follow a Gaussian distribution. A great advantage of Gaussian network is that they need $\mathrm{O}(\mathrm{n} 2)$ parameters to model a complete graph. Their work showed how classifiers, supported by the Bayesian network paradigm, can be adapted to deal with continuous variables without discretizing them. In addition, two novel classifier learning algorithms were introduced. The presented learning algorithms were ordered and grouped according to their structural complexity: from the simplest naive Bayes structures to k-dependence Bayesian classifiers and semi naive Bayes. Moreover, for each structure a filter and wrapper approaches were presented. All these classifiers were empirically evaluated using the Brier score and the predictive accuracy. The obtained results with both scores suggested that semi naive Bayes is the best classifier. 


\section{Comparison of Algorithms}

\begin{tabular}{|c|c|c|c|c|c|c|c|c|c|}
\hline pach & Selection & Crossover & Mutation & $\begin{array}{l}\text { Fitness } \\
\text { Criteria }\end{array}$ & $\begin{array}{l}\text { Population } \\
\text { size }\end{array}$ & $\begin{array}{l}\text { Stopping } \\
\text { criteria }\end{array}$ & Coding & Feature & Future work \\
\hline $\begin{array}{l}\text { GA with } \\
\text { Threshold } \\
{[34]}\end{array}$ & $\begin{array}{l}\text { Tournament } \\
\text { Strategy }\end{array}$ & $P_{c}=0.9$ & $\begin{array}{l}P_{\mathrm{m}}=0.05 \\
\text { (follows } \\
\text { decaying } \\
\text { exponential } \\
\text { function) }\end{array}$ & $\begin{array}{l}\text { Normalized } \\
\text { histogram } \\
\text { function }\end{array}$ & $\begin{array}{l}\mathrm{N}_{\text {size }}=20 \\
\text { (between } \\
\text { two } \\
\text { correspondi } \\
\text { ng gray } \\
\text { values) }\end{array}$ & $\begin{array}{l}\text { Until } \\
\text { elements of } \\
\text { respective } \\
\text { classes are } \\
\text { equally fit }\end{array}$ & $\begin{array}{l}\text { RGB } \\
\text { component } \\
s\end{array}$ & $\begin{array}{l}\text { Peaks at gray } \\
\text { value= } 91,148 \\
\text { Threshold= } \\
117\end{array}$ & $\begin{array}{l}\text { To address two } \\
\text { class images wit } \\
\text { noises and } \\
\text { images requirin } \\
\text { multiple } \\
\text { thresholds }\end{array}$ \\
\hline $\begin{array}{l}\text { Adaptive } \\
\text { GA[39] }\end{array}$ & $\begin{array}{l}\text { Set of } 20 \\
\text { features }\end{array}$ & $P_{c}=0.8$ & $\begin{array}{l}P_{m}=0.01 \\
\text { If fitness } \\
\text { value is not } \\
\text { improved } \\
\text { for } 3 \\
\text { generations } \\
P_{m}=0.02\end{array}$ & $\begin{array}{l}\text { MDLP based } \\
\text { Fitness } \\
\text { function } \\
\end{array}$ & $N_{\text {size }}=100$ & $\begin{array}{l}\text { Error rate of } \\
\text { best set of } \\
\text { features } \\
\text { selected is } \\
\text { below } \\
\text { threshold or } \\
\text { mutation rate } \\
\text { is increased } \\
\text { above } 0.09\end{array}$ & $\begin{array}{l}\text { Binary } \\
\text { coding }\end{array}$ & \begin{tabular}{|l|} 
Adaptive GA \\
that \\
automatically \\
adapt the \\
parameters(cro \\
ssover/mutatio \\
n) based on its \\
performance
\end{tabular} & $\begin{array}{l}\text { To extend this } \\
\text { approach to } \\
\text { additional } \\
\text { features and } \\
\text { more complex } \\
\text { background } \\
\text { clutter. }\end{array}$ \\
\hline $\begin{array}{l}\text { Parallel } \\
\text { GA[11] }\end{array}$ & $\begin{array}{l}\text { Fitness } \\
\text { Proportional } \\
\text { model }\end{array}$ & $P_{c}=0.6$ & $P_{m}=0.1$ & $\begin{array}{l}\text { Distribution } \\
\text { function }\end{array}$ & $N_{\text {size }}=100$ & 250 iterations & $\begin{array}{l}\text { Integer } \\
\text { (Chromoso } \\
\text { me } \\
=5)\end{array}$ & $\begin{array}{l}\text { Incorporates } \\
\text { migration } \\
\text { engthoperation }\end{array}$ & $\begin{array}{l}\text { To solve the } \\
\text { occlusion } \\
\text { problem } \\
\text { completely }\end{array}$ \\
\hline $\begin{array}{l}\text { Dynamic GA } \\
\text { Based } \\
\text { Clustering } \\
\text { (GADCIS) } \\
{[32]} \\
\end{array}$ & $\begin{array}{l}\text { Roulette } \\
\text { Wheel } \\
\text { selection }\end{array}$ & $\begin{array}{l}\mathrm{P}_{\mathrm{c}}=0.9 \\
\text { One point } \\
\text { Crossover }\end{array}$ & $P_{m}=0.1$ & $F=1 / M$ & $N_{\text {size }}=30$ & $\begin{array}{l}20 \text { iterations } \\
\text { Elitism }\end{array}$ & $\begin{array}{l}\text { RGB } \\
\text { component }\end{array}$ & $\begin{array}{l}\text { No prior } \\
\text { knowledge } \\
\text { required }\end{array}$ & $\begin{array}{l}\text { To be used in } \\
\text { various fields } \\
\text { like MRI and } \\
\text { satellite images }\end{array}$ \\
\hline $\begin{array}{l}\text { GA } \\
\text { (for } \\
\text { Adaptive } \\
\text { Image } \\
\text { Segmentati } \\
\text { on) }[30] \\
\end{array}$ & $\begin{array}{l}\text { Threshold- } \\
\text { crossing } \\
\text { selection } \\
\text { criteria }\end{array}$ & $\begin{array}{l}\mathrm{P}_{\mathrm{c}}=0.6 \\
\text { One point } \\
\text { crossover }\end{array}$ & $P_{m}=0.001$ & $\begin{array}{l}\text { Fitness value } \\
\text { ranging } \\
\text { around 500- } \\
570\end{array}$ & $\mathrm{~N}_{\text {size }}=200$ & 200 iteration & $\begin{array}{l}\text { Computer } \\
\text { assisted } \\
\text { segmentati } \\
\text { on }\end{array}$ & $\begin{array}{l}\text { GA repeatedly } \\
\text { converges to } \\
\text { almost same } \\
\text { fitness values }\end{array}$ & $\begin{array}{l}\text { To let GA itself } \\
\text { dynamically } \\
\text { select } \\
\text { segmentation } \\
\text { algorithm }\end{array}$ \\
\hline $\begin{array}{l}\text { Simple GA } \\
{[37]}\end{array}$ & $\begin{array}{l}\text { Roulette } \\
\text { wheel } \\
\text { selection }\end{array}$ & $\begin{array}{l}\mathrm{P}_{\mathrm{c}}=0.2 \\
\text { Two-point } \\
\text { crossover }\end{array}$ & $\begin{array}{l}P_{m}=0.1 \\
\text { Dynamic } \\
\text { mutation } \\
\text { operator }\end{array}$ & $\begin{array}{l}\text { Fuzzy fitness } \\
\text { function }\end{array}$ & $N_{\text {size }}=60$ & 300 iterations & $\begin{array}{l}\text { Gene } \\
\text { integer } \\
\text { type }\end{array}$ & $\begin{array}{l}\text { Efficient } \\
\text { without the } \\
\text { need for critical } \\
\text { parameters or } \\
\text { threshold } \\
\text { values }\end{array}$ & $\begin{array}{l}\text { To use prior } \\
\text { knowledge }\end{array}$ \\
\hline $\begin{array}{l}\text { Genetic c- } \\
\text { Means } \\
\text { Clustering } \\
\text { (GCMA) [36] }\end{array}$ & - & $\begin{array}{l}\mathrm{P}_{\mathrm{c}}=0.8 \\
\text { One point } \\
\text { crossover }\end{array}$ & $P_{m}=0.05$ & $\begin{array}{l}\text { Inverse of } \\
\text { MSE }\end{array}$ & $\begin{array}{l}N_{\text {size }}=\text { no. of } \\
\text { clusters }\end{array}$ & $\begin{array}{l}\text { Until the } \\
\text { stored string } \\
\text { approaches } \\
\text { an optimum }\end{array}$ & $\begin{array}{l}\text { Integer or } \\
\text { floating } \\
\text { point }\end{array}$ & $\begin{array}{l}\text { Develops a } \\
\text { hybrid } \\
\text { algorithm } \\
\text { combining } \\
\text { CMA and GA }\end{array}$ & $\begin{array}{l}\text { To measure } \\
\text { effects of local } \\
\text { optimality on } \\
\text { other color } \\
\text { image } \\
\text { segmentation } \\
\text { techniques }\end{array}$ \\
\hline
\end{tabular}




\begin{tabular}{|c|c|c|c|c|c|c|c|c|c|}
\hline $\begin{array}{l}\text { GAHSI } \\
\text { algorithm } \\
\text { [33] }\end{array}$ & $\begin{array}{l}\text { Local } \\
\text { tournament } \\
\text { selection } \\
\text { over roulette } \\
\text { wheel } \\
\text { method }\end{array}$ & $\begin{array}{l}\text { Single point } \\
\text { crossover } \\
\mathrm{P}_{\mathrm{c}}=0.8\end{array}$ & $P_{m}=0.03$ & $\begin{array}{l}\text { Weighted } \\
\text { average } \\
\text { function }\end{array}$ & $N_{\text {size }}=48$ & $\begin{array}{l}\text { Threshold } \\
\text { technique } \\
\text { based on } \\
\text { UTOPIA } \\
\text { parameter } \\
\text { set, if it failed } \\
\text { for } \\
\text { consecutive } 5 \\
\text { times } \\
\end{array}$ & Binary & $\begin{array}{l}\text { Overcomes the } \\
\text { effects of } \\
\text { variable } \\
\text { outdoor } \\
\text { lighting } \\
\text { conditions with } \\
\text { an acceptable } \\
\text { error range. }\end{array}$ & $\begin{array}{l}\text { To further } \\
\text { improve } \\
\text { segmentation } \\
\text { robustness as } \\
\text { well as GA } \\
\text { coding and } \\
\text { operators in } \\
\text { variable } \\
\text { conditions }\end{array}$ \\
\hline $\begin{array}{l}\text { Unsupervise } \\
\text { d clustering } \\
\text { using } \\
\text { GA[15] }\end{array}$ & $\begin{array}{l}\text { Minimum } \\
\text { distance } \\
\text { criteria }\end{array}$ & $P_{c}=0.95$ & $P_{m}=0.85$ & $\begin{array}{l}\text { K-nearest } \\
\text { neighbour } \\
\text { Rule }\end{array}$ & $N_{\text {size }}=50$ & $N_{p o p}=10000$ & Binary & $\begin{array}{l}\text { Clustering of } \\
\text { small regions in } \\
\text { color feature } \\
\text { space }\end{array}$ & $\begin{array}{l}\text { To study the } \\
\text { cluster relations } \\
\text { (clouds of points) } \\
\text { for each } \\
\text { segmentation } \\
\text { problem }\end{array}$ \\
\hline $\begin{array}{l}\text { ISODATA } \\
\text { Algorithm } \\
\text { [35] }\end{array}$ & $\begin{array}{l}\text { Roulette } \\
\text { wheel } \\
\text { method }\end{array}$ & $\begin{array}{l}\text { Cluster } \\
\text { Centre } \\
\text { replacement } \\
\text { Method } \\
\mathrm{P}_{\mathrm{C}}=0.6\end{array}$ & $\begin{array}{l}\text { Random } \\
\text { substitution } \\
\mathrm{P}_{\mathrm{m}}=0.1\end{array}$ & $\begin{array}{l}\text { Pbjective } \\
\text { Function }\end{array}$ & $N_{\text {size }}=90$ & $\begin{array}{l}\text { Stability of } \\
\text { fitness values } \\
\text { for } 20 \\
\text { iterations }\end{array}$ & $\begin{array}{l}\text { Bit level } \\
\text { coding }\end{array}$ & $\begin{array}{l}\text { Uses an ANN } \\
\text { called } \\
\text { Kohonen's } \\
\text { sOM and } \\
\text { hybrid genetic } \\
\text { algorithm } \\
\text { (HGA) }\end{array}$ & $\begin{array}{l}\text { To use a } \\
\text { knowledge base } \\
\text { that contains the } \\
\text { cluster centres of } \\
\text { previous } \\
\text { segmentation on } \\
\text { different satellite } \\
\text { images. }\end{array}$ \\
\hline
\end{tabular}

\section{FUTURE WORK}

After reviewing a set of papers on image segmentation we found that the algorithms implemented in these papers still have some areas of improvement. In paper [11], the problem of occlusion was eliminated partially. Amiya Halder, Nilavra Pathak[32] promoted the application of genetic algorithm on satellite and MRI images to demonstrate its wide applicability. [39] proved through experiments good performance of GA and plans to further extend its applicability. In [36] emphasis is on further advancing the use of genetic algorithm to improve color image segmentation techniques. [37] promoted to use prior knowledge based segmentation. GHASI [33] revealed that in variable lighting conditions, the genetic algorithm-based, supervised color image segmentation in HSI color space is an effective approach but further improvement in segmentation robustness can be achieved. [15][35] considers to improve the potential of GA in future, by providing it with useful information like clustering relationships for segmentation problem [15] and providing a knowledge base for improved segmentation of satellite images[35]. The prospects and problems revealed in above cited papers can be resolved by integrating the segmentation processing with a classification process to provide the required semantic information and thus have appropriate results. The wrapper framework for feature selection wraps the selection process inside the classifier in order to determine the best possible segmentation. Besides wrapper, Genetic Algorithm is proposed to provide optimal solution from large amount of multidimensional data in minimum possible time to achieve all the prospects mentioned in [37][15][30][33][35][36]. Therefore by taking together the features of wrapper framework and genetic algorithm our focus is to take the work of above mentioned papers one step ahead and acquire proficient segmented image.

\section{RESULT}

In accordance with the future work to improve segmentation results using some prior knowledge, we implemented classification algorithm on the input image to enhance the results of the color based segmentation using genetic algorithm. In our classification algorithm we classified the RGB image into $n$ classes, each class representing a color. The obtained value of number of classes is taken as input to image segmentation. Our proposed algorithm of image segmentation using genetic algorithm is as stated below.

1. Repeat step 2 to 4 till fixed number of generations.

2. Randomly generate the cluster set.

3. Each pixel of the image is labelled to the generated cluster number using Euclidean distance criterion.

4. Evaluate the fitness value.

5. The input image is segmented over the cluster set that has maximum fitness value. 
We compared the results of our algorithm with the results obtained by using DCPSO using $v$ and SNOB [78]. As per our proposed algorithm, implemented in MATLAB R2007b, certain outcomes are as follows:

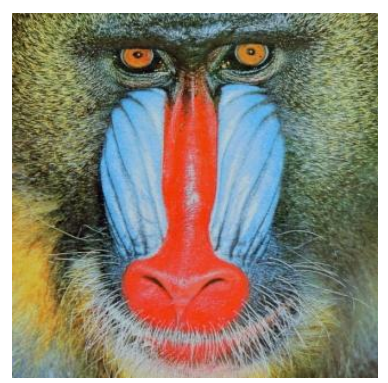

True RGB Image(Mandril.jpg)

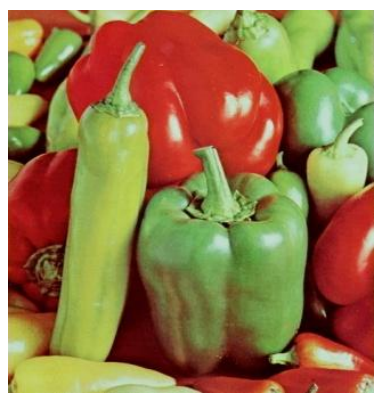

True RGB Image(Peppers.jpg)

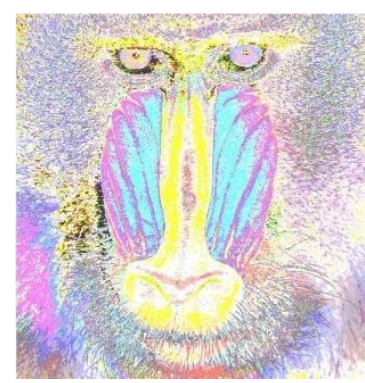

Segmented Image

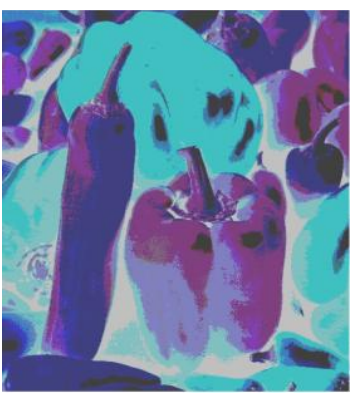

Segmented Image
Table 1: Results

\begin{tabular}{|l|l|l|l|l|}
\hline Image & $\begin{array}{l}\text { Optimal } \\
\text { range }\end{array}$ & $\begin{array}{l}\text { DCPSO } \\
\text { using v }\end{array}$ & SNOB & $\begin{array}{c}\text { Proposed } \\
\text { method }\end{array}$ \\
\hline Mandrill & 5 to 10 & 6 & 39 & 6.32 \\
\hline Lenna & 5 to 10 & 6.5 & 31 & 5.47 \\
\hline Peppers & 6 to 10 & 6.25 & 42 & 6.13 \\
\hline Jet & 5 to 7 & 5.35 & 22 & 4.39 \\
\hline
\end{tabular}

\section{CONCLUSION}

The main aim of this paper was to study various researches in the field of image segmentation and to categorise them with respect to the image segmentation process. After analysing a large number of papers, we identified major trends in the development of image segmentation. We also reviewed wrapper framework based on Gaussian networks that deals with continuous variables without discretizing them. However, since the level of sophistication of newly proposed algorithms is constantly increasing it is very difficult to predict which approach will dominate the field of image analysis. The design of a segmentation system must address issues of efficiency in addition to that of effectiveness. There is no universal theory on image segmentation till now.

They are strongly application dependent, in other words, there are no general algorithms that are good for all images.

An important advantage of a genetic methodology lies in that the fitness function provides a natural means to model the uncertainty in an image. We have successfully used prior knowledge i.e. color through the classification algorithm and used it to segment the image efficiently on the basis of classes defined. The accuracy of the classifier in the classification algorithm can be measured to further improve the segmentation. Subsequently, image segmentation results can be utilized in feature extraction and object recognition phases of image processing and computer vision.

\section{ACKNOWLEDGMENTS}

We take this opportunity to express our profound gratitude and deep regards to our guide, Professor Vijai Singh, for his exemplary guidance, monitoring and constant encouragement throughout the course of this project.

\section{REFERENCES}

[1] W. Frei, C. Chen, "Fast Boundary Detection: A Generalization and New Algorithm," IEEE Trans. Computers, vol. C-26, no. 10, pp. 988-998, Oct. 1977.

[2] J. Canny, "A computational approach to edge detection," IEEE Trans. Pattern Analysis and Machine Intelligence, Vol. 8, No. 6, pp. 679-698, Nov. 1986.

[3] W. K. Pratt, "Digital Image Processing". New York, NY: Wiley-Interscience, 1991, pp. 491-556.

[4] JL. Vincent, "Morphological gray scale reconstruction in image analysis: Applications and efficient algorithms", IEEE Trans. Image Processing, vol. 2, 1993.

[5] S.Beucher, F.Meyer. "The morphological approach to segmentation: The watershed transform", in Mathematical Morphology Image Processing, E. R. Dougherty, Ed. New York Marcel Dekker, 1993, vol. 12, pp. 433-481.

[6] B. Bhanu, S. Lee, J. Ming. "Adaptive image segmentation using a genetic algorithm", In IEEE Transactions on Systems, Man and Cybernetics, volume 25, pages 1543-1567, Dec 1995.

[7] A. N. Moga, M. Gabbouj, "Parallel image component labeling with watershed transformation", IEEE Trans. Pattern Anal. Machine Intell. vol. 19, pp. 441-450. J. M Gauch, "Image segmentation and analysis via multi-scale gradient watershed hierarchies", IEEE Trans Image Processing, vol. 8, pp. 69-79, 2000.

[8] B. Bhanu, J. Peng., "Adaptive integrated image segmentation and object recognition", In IEEE Transactions on Systems, Man and Cybernetics, Part C, volume 30, pages 427-441, November 2000.

[9] O. F. Olsen, M. Nielsen, "Multi-scale gradient magnitude watershed segmentation", in ICIAP' 97-9th Int Conference on Image Analysis and Processing, ser. Lecture Notes in Computer Science. Berlin, Germany: Springer-Verlag, 2001, vol. 1310, pp. 6-13.

[10] R. C. Gonzalez and R. E. Woods, "Digital Image Processing". Upper Saddle River, NJ: Prentice-Hall, 2001, pp. 572-585.

[11] Tianzi Jiang, Faguo Yang, Yong Fan, David J. Evans., "A Parallel Genetic Algorithm for Cell Image Segmentation", August 2001.

[12] Gonzalez and Woods, "Digital image processing", 2nd Edition, prentice hall, 2002.

[13] Kenneth R. Castelman, "Digital image processing", Tsinghua Univ Press, 2003. 
[14] Michael Farmer, "A wrapper-based approach to image segmentation and classification", 2004.

[15] Vitorino Ramos, Fernando Muge., "Image Colour Segmentation by Genetic Algorithms", 2004.

[16] Leo Grady and Eric L. Schwartz "Isoperimetric Graph Partitioning for Image Segmentation", Pattern Analysis and Machine Intelligence, IEEE Transactions.

[17] M.E. Farmer and D. Shugars. "Application of genetic algorithms for wrapper-based image segmentation and classification", In IEEE Congress on Evolutionary Computation, pages 1300-1307, July 2006.

[18] Keri Woods. "Genetic Algorithms: Colour Image Segmentation Literature Review", July 24, 2007.

[19] Aritz P'erez, Pedro Larra naga, Innaki Inza., "Supervised Classification with Gaussian Networks: Filter and Wrapper Approaches", 24th August, 2007.

[20] Jun Zhang, Kitakyushu Jinglu Hu. "Computer Science and Software Engineering",International Conference, 2008.

[21] N. Senthilkumaran, R. Rajesh. "Edge Detection Techniques for Image Segmentation - A Survey", Proceedings of the International Conference on Managing Next Generation Software Applications (MNGSA-08), 2008, pp.749-760.

[22] Michael Farmer. "Application of the wrapper framework for image object detection", December, 2008.

[23] Du Gen-yuan, Miao Fang, Tian Sheng-li, Guo Xi-rong. "Remote Sensing Image Sequence Segmentation Based on the Modified Fuzzy C-means", Journal of Software, Vol. 5, No. 1, PP.28-35, 2009.

[24] Utkarsh Kumar Shah and Prof. A. Mukherjee, "BTP REPORT Image segmentation by region growing", April $15,2010$.

[25] ${ }^{1}$ Mandeep Kaur, ${ }^{2}$ Gagandeep Jindal, 1,2 Dept. of CEC, Landran, Mohali, Punjab, India.

[26] Prateek Gupta, Sargam Saxena, Sonali Singh, Saumya Dhami, Vijai Singh, "Color Image Segmentation: A State of the Art Survey", International Journal of Computational Intelligence Research ISSN 0973-1873 Volume 8, Number 1 (2012), pp. 17-25 @ Research India Publications.

[27] Shilpa Kamdi1, R.K.Krishna2. 1, 2-Rajiv Gandhi College of Engineering, Research and Technology, "Image Segmentation and Region Growing Algorithm", Feb 1, 2012.

[28] Suryakant, Neetu Kushwaha. "Edge Detection using Fuzzy Logic in Matlab," ISSN: 2277 128X, Volume 2, Issue 4, April, 2012.

[29] Prerna Pachunde, Prof.Vikal.R.Ingle, Prof. Dr Mahindra. A. Gailwad. "Segmentation of Color Images Using Genetic Algorithms: A Survey", July-Aug. 2012

[30] S. Cagnoni, A. B. Dobrzeniecki, R. Poli, J. C. Yanch. "Genetic algorithm-based interactive segmentation of 3D medical images", 1999.

[31] Zhenyu Wu and Richard Leahy, "An Optimal Graph theoretic approach to data clustering: Theory and its Application to Image Segmentation", IEEE Transactions on pattern analysis and machine intelligence, Vol. 15 No. 11, Nov. 1993.

[32] Amiya Halder, Nilavra Pathak. "An Evolutionary Dynamic Clustering Based Color Image Segmentation”, International Journal of Image Processing (IJIP) Volume (4): Issue (6).

[33] L. Tang, L. Tian, B. L. Steward. "Color Image Segmentation with Genetic Algorithm For In-Field Weed Sensing", September, 2001.

[34] P. Kanungo, P. K. Nanda, U. C. Samal. "Image Segmentation Using Thresholding and Genetic Algorithm", Volume 1, 2006.

[35] Mohamad Awad, Kacem Chehdi, Ahmad Nasri. "Multicomponent Image Segmentation Using a Genetic Algorithm and Artificial Neural Network", ieee geoscience and remote sensing letters, VOL. 4, NO. 4, October, 2007.

[36] Paul Scheunders., "A Genetic C-Means Clustering Algorithm Applied to Color Image Quantization".

[37] Dae N. Chun, Hyun S. Yang, "Robust Image Segmentation using Genetic Algorithm with a Fuzzy Measure", Pattern Recognition, Vol 29, No. 7,pp 1195$1211,1996$.

[38] Francisco J. Estrada \& Allan D. Jepson, "Benchmarking Image Segmentation Algorithms", Int J Comput Vis (2009) 85: 167-181, DOI 10.1007/s11263-009-0251-z, 28 May 2009.

[39] Bir Bhanu, Yingqiang Lin, "Genetic Algorithm based feature selection for target detection in SAR images", Image and Vision Computing 21 (2003) 591-608.

[40] Mr. Salem Saleh Al-amri, Dr. N.V. Kalyankar and Dr. Khamitkar S.D, "Image Segmentation By Using Edge Detection", (IJCSE) International Journal on Computer Science and Engineering Vol. 02, No. 03, 2010, 804-807.

[41] C. K. Chow and T. Kaneko, "Boundary detection of radiographic images by a threshold method", in Proceedings, ZFZP Congress 71, pp. 130-134.

[42] C. K. Chow and T. Kaneko, "Automatic boundary detection of left ventricle from cineangiograms", Comput. Biomed. Res. 5, 1972, 338-410.

[43] D. Mason, I. J. Lauder, D. Rutoritz, and G. Spowart, "Measurement of C-Bands in human Chromosomes", Comput. Biol. Med. 5, 1975, 179-201.

[44] Y. H. Katz, "Pattern recognition of meteorological satellite cloud photography, Proceedings", Third Symposium on Remote Sensing of Environment, 1965, pp 173-214

[45] J. S. Weszka and A. Rosenfeld, "Threshold Selection 4", TR-336, University of Maryland Computer Science Center, 1974.

[46] J. S. Weszka, R. N. Nagel, and A. Rosenfeld, "A threshold selection technique", IEEE Trans. Comput C23, 1974, 1322-1326.

[47] S. Watanabe, and the CYBEST group, "An automated apparatus for cancer processing CYBEST", Computer Vision Graphics Image Process. 3, 1974, 350-358.

[48] J. S. Weszka, J. A. Veretuon, and A. Rosenfeld, "A Technique for Facilitating Threshold Selection for Objects Extraction from Digital Pictures", TR-243, University of Maryland Computer Science Center, 1973. 
[49] J. S. Weszka and A. Rosenfeld, "Histogram modification for threshold selection", IEEE Trans. Systems Man Cybernet. SMC-9, 1979, 38-51.

[50] Rolf Adams and Leanne Bischof, "Seeded Region Growing", IEEE Transactions on Pattern Analysis and Machine Intelligence, VOL. 16, NO. 6, June 1994. 18.

[51] R.Sridevi, K. Krishnaveni, S. Navaneethakrishnan, "A novel watershed image segmentation technique using graceful labelling", International Journal of Mathematics and Soft Computing Vol.3, No.1. (2013), 69 - 78.

[52] Mark Dow and Robert, Beverly Lewis, "An Edge Based Image Segmentation Method", ISMRM, 2004.

[53] S. K. Pal et al., "A Review on Image Segmentation Techniques, Pattern recognition”, Vol. 29 (1993), 12771294.

[54] H. S. Prasantha, Dr. Shashidhara.H.L, Dr. K. N. B. Murthy, Madhavi Lata.G, "Medical Image Segmentation", (IJCSE) International Journal on Computer Science and Engineering Vol. 02, No. 04, 2010, 1209-1218.

[55] Mantas Paulinas, Andrius Ušinskas, "A Survey Of Genetic Algorithms Applications For Image Enhancement and Segmentation", Electronic Systems Department, Faculty of Electronics, Vilnius Gediminas Technical University.

[56] N.R. Harvey, S. Marshall, "The design of different classes of morphological filter using genetic algorithms", IEE fifth international conference on image processing and its applications, 1995, $227-231$.

[57] M. Mitchell, "An introduction to genetic algorithms", The MIT Press, 1996, 208.

[58] H. Peng, F. Long, Z. Chi, and W. Su, "A hierarchical distributed genetic algorithm for image segmentation", in Proc. 2000 Congress on Evolutionary Computation, vol. 1, 2000, pp. 272-276.

[59] S.M. Bhandarkar and H. Zhang., "Image segmentation using evolutionary computation", IEEE Transactions on Evolutionary Computation, volume 3, pages 1-21, April 1999.

[60] S.E. Papadakis, P. Tzionas, V.G. Kaburlasos, J.B. Theocharis., "A genetic based approach to the type I structure identification", Informatica, 2005, Vol.16, No. $3,365-382$.

[61] H.P Schwefel, G. Rudolph, "Contemporary evolution strategies", Advances in artificial life, 1995, 893 - 907.

[62] P. Andrey, "Selectionist relaxation: Genetic algorithms applied to image segmentation", Image and Vision Computing, vol. 17, pp. 175-187, 1999.

[63] D. L. Swets, B. Punch, and J. Weng, "Genetic algorithms for object recognition in a complex scene", Proc. 1995 Int. Conf. Image Processing (ICIP'95), 1995.

[64] K. I. Laws, "The phoenix image segmentation system: Description and evaluation", SRI AI Center, 1982.

[65] R. Q. Feitosa, G. A. O. P. Costa, and T. B. Cazes, "A genetic approach for the automatic adaptation of segmentation parameters", Proc. OBIA06, 2006.
[66] P. Zingaretti, G. Tascini, and L. Regini, "Optimising the colour image segmentation", Proc. VIII Convegno dell Associazione Italiana per Intelligenza Artificiale, 2002.

[67] G. Pignalberi, R. Cucchiara, L. Cinque, and S. Levialdi, "Tuning range image segmentation by genetic algorithm", EURASIP Journal on Applied Signal Processing, vol. 8, pp. 780-790, 2003.

[68] D. N. Chun and H. S. Yang, "Robust image segmentation using genetic algorithm with a fuzzy measure", Pattern Recognition, vol. 29, no. 7, pp. 11951211, 1996.

[69] M. Gong and Y. H. Yang, "Genetic-based multiresolution color image segmentation", Proc. Vision Interface 2001, pp. 141-148.

[70] B. Bhanu and J. Peng, "Adaptive integrated image recognition and segmentation", IEEE Trans. on Systems, Man, and Cybernetics-Part C: Applications and Reviews, vol. 30, no. 4, pp. 427-441, Nov. 2000.

[71] J. Peng and B. Bhanu, "Closed-loop object recognition using reinforcement learning", IEEE Trans. on Pattern Analysis and Machine Intelligence, VOL. 20, NO. 2, 1998.

[72] Michael E. Farmer and Anil K. Jain, "A Wrapper-Based Approach to Image Segmentation and Classification", IEEE Transactions On Image Processing, VOL. 14, NO. 12, December2005.

[73] Ron Kohavi1 and George H. John2, "The Wrapper Approach", 1- Data mining and Visualisation, 2- Data mining.

[74] Jinsong Leng, Craig Valli, Leisa Armstrong, “A Wrapper-Based Feature Selection for Analysis of Large Data Sets", 2010 3rd International Conference on Computer and Electrical Engineering (ICCEE 2010).

[75] Hossein Mobahi, Shankar Rao, Allen Yang, Shankar Sastry and Yi Ma, "Segmentation of Natural Images by Texture and Boundary Compression", International Journal of Computer Vision (IJCV), 95 (1), pg. 86-98, Oct. 2011

[76] Shankar Rao, Hossein Mobahi, Allen Yang, Shankar Sastry and Yi Ma, "Natural Image Segmentation with Adaptive Texture and Boundary Encoding", Proceedings of the Asian Conference on Computer Vision (ACCV) 2009, H. Zha, R.-i. Taniguchi, and S. Maybank (Eds.), Part I, LNCS 5994, pp. 135--146, Springer.

[77] M. Mohamed Sathik, K. Senthamarai Kannan and Y. Jacob Vetha Raj, "HYBRID JPEG COMPRESSION USING EDGE BASED SEGMENTATION", Signal \& Image Processing: An International Journal(SIPIJ) Vol.2, No.1, March 2011.

[78] Mahamed G. H. Omran, "Particle Swarm Optimization Methods for Pattern Recognition and Image Processing", Submitted in partial fulfillment of the requirements for the degree Philosophiae Doctor in the Faculty of Engineering, Built Environment and Information Technology, University of Pretoria, Pretoria, November 2004. 